\title{
Gravity from Quantum Information
}

\author{
Jae-Weon Lee* \\ Department of energy resources development, Jungwon University, \\ 5 dongburi, Goesan-eup, Goesan-gun Chungbuk Korea 367-805 \\ Hyeong-Chan $\operatorname{Kim}^{\dagger}$ \\ School of Liberal Arts and Sciences, Korea National University of Transportation, Chungju, 380-702, Korea \\ Jungjai Le€ \\ Department of Physics, Daejin University, Pocheon, Gyeonggi 487-711, Korea
}

(Dated: October 22, 2018)

\begin{abstract}
It is suggested that the Einstein equation can be derived from Landauer's principle applied to an information erasing process at a local Rindler horizon and Jacobson's idea linking the Einstein equation with thermodynamics. When matter crosses the horizon, the information of the matter disappears and the horizon entanglement entropy increases to compensate the entropy reduction. The Einstein equation describes an information-energy relation during this process, which implies that entropic gravity is related to the quantum entanglement of the vacuum and has a quantum information theoretic origin.
\end{abstract}

PACS numbers: 98.80.Cq, 98.80.Es, 03.65.Ud

\footnotetext{
* scikid@gmail.com

† hyeongchan@gmail.com

$\ddagger$ jjlee@daejin.ac.kr
} 


\section{INTRODUCTION}

The Einstein equation express a relation between matter and the spacetime geometry that the matter disturbs. Despite of the clear geometric meaning of the Einstein tensor, the origin of this relation still remains a mystery. In 1995, Jacobson suggested an interesting idea that the Einstein equation [1, 2] actually describes the equation of state (or, the first law of thermodynamics) at local Rindler horizons. Recently, Verlinde brought us a remarkable new idea [3] linking gravitational force to entropic force. He derived the Newton's equation and the Einstein equation from the connection. Padmanabhan also proposed that the Einstein equation is from the equipartition law [4].

One can ask why there is such a surprising relationship between thermodynamics, especially entropy, and gravity. In this paper, we try to answer to this fundamental question by considering quantum information erasure in a curved spacetime. In a series of works [5- 7] we emphasized the quantum informational nature of gravity. For example, we suggested that dark energy responsible for the cosmic accelerating expansion is related to the quantum entanglement of the vacuum fluctuation [5] or erasure of quantum information at a cosmic horizon [8, 9]. A cosmic horizon with a radius $R_{h} \sim O\left(H^{-1}\right)$ has a kind of thermal energy $E_{h} \propto T_{h} S_{h} \propto R_{h}$ associated with its holographic entropy $S_{h} \propto R_{h}^{2}$, and this thermal energy has an information theoretic origin. Here $H$ is the Hubble parameter. (Recently, there appear similar suggestions based on the Verlinde's idea [10 13].) To be specific we identified $S_{h}$ as an entanglement entropy $S_{E n t}$ associated with the erased vacuum information outside the horizon, and $T_{h} \propto 1 / R_{h}$ as the Hawking-Gibbons temperature of the horizon. Then, it was straight forward to get a horizon energy density $\rho_{h} \sim E_{h} / R_{h}^{3} \sim N_{s} M_{P}^{2} / R_{h}^{2}$ which can be interpreted as a holographic dark energy density [14]. Here, Planck mass $M_{P}$ is a UV-cutoff and $N_{s}$ is the number of spin degree of freedom of quantum fields. With reasonable input parameters our dark energy model gives the energy density and the equation of state for dark energy comparable to the observational data [5]. (This dark energy could be also regarded as the energy of cosmic Hawking radiation [9].)

Interestingly, in principle, one can explicitly obtain $S_{E n t}$ for the vacuum state using quantum field theory. In [15] $S_{E n t}$ for a spherical region is obtained by partial tracing a ground state of discretized quantum fields. Using a similar approach we also derived the first law of black hole thermodynamics from the second law of thermodynamics [6] and obtained a discrete black hole mass formula. These works are based on the Landauer's principle in quantum information theory and the holographic principle. As a variant of the second law of thermodynamics, Landauer's principle states that to erase $N$ bits of information of a system irreversibly at least $k_{B} N$ entropy of a bath should be increased and at least $k_{B} N T$ energy should be consumed, where $k_{B}$ is the Boltzman's constant and $T$ is the temperature of the thermal bath contacting with the system. For a black hole and the universe their causal horizons play roles of the bath as well as an information barrier. We suggested that the horizons have thermal energy $E_{h}$ related to the information erasing at the horizons given by

$$
d E_{h}=k_{B} T_{h} d S_{h}
$$

where $T_{h}$ is a horizon temperature and $d S_{h}$ is a horizon entropy change due to the information erasing. We identified this energy as the origin of dark energy or black hole mass. Thus, one can see that this energy is very similar to the equipartition energy for the entropic gravity [3, 4]. (Strictly speaking, the relation in Eq.(1) is more likely the Clausius relation, however we call it the first law by convention.)

All our results above imply that there is an intrinsic relationship between quantum information and gravity. This can be seen as another realization of the famous slogan in quantum information community: "It from Bit!" Along this line in this paper we suggest that the Einstein equation itself can be derived by considering quantum information theory applied to Rindler horizons of a given spacetime. Our work is also based on the work by Jacobson linking the first law of thermodynamics to the Einstein equation. In Sec. II, we study the relation between entanglement and entropic gravity. In Sec. III, we derive the Einstein equation from information loss. In Sec. IV, we summarize and discuss the results.

\section{ENTANGLEMENT AND ENTROPIC GRAVITY}

In this section, we show that the entropic gravity can be related to the quantum entanglement and Landauer's principle applied to causal horizons.

The quantum entanglement is one of the key concepts of quantum information theory allowing useful quantum information processes such as quantum key distribution. It is a quantum nonlocal correlation which can not be described by a classical correlation. The entanglement entropy $S_{E n t}$ is a good measure of entanglement for pure states such as the vacuum. It is the von Neumann entropy $S_{E n t}=-\operatorname{Tr}\left(\rho_{A} \ln \rho_{A}\right)$ associated with the reduced density matrix $\rho_{A} \equiv \operatorname{Tr}_{B} \rho_{A B}$ of a bipartite system $A B$ described by a full density matrix $\rho_{A B}$. Why can we regard $S_{E n t}$ of the quantum field vacuum as $S_{h}$ ? For a causal horizon playing a role of information barrier, it is natural to divide 
the system into two subsystems A and B - inside and outside the horizon - and to trace over one of the two regions to obtain the entanglement entropy of the horizon. Thus, $S_{E n t}$ is ideal for $S_{h}$, when there is a causal horizon.

Using Landauer's principle, we calculated black hole mass increase due to absorbtion of a test particle with energy $\delta E$ in the context of quantum information theory in $[\underline{6}$. To the observer outside the black hole, this corresponds to the erasure of $\delta S$ bits of information by the thermal bath of the event horizon. In this case the Landauer's principle demands that the entropy of the black hole increases at least by $\delta S$ during this process. Assuming this erasing process is optimal, we obtained the increase of the black hole mass $\delta M_{B H}=\delta E=k_{B} T \delta S$, which looks like the first law of black hole thermodynamics. Here, $T$ is the Hawking temperature for the black hole. In the work, to calculate the lost information of the particle crossing the horizon we assumed an information erasing process similar to that considered by Song and Winstanley [16]. They derived a generalized second law for black hole thermodynamics from the view point of quantum information theory, especially by applying the Landauers principle. They considered a small quantum system falling into the black hole with the Hawking temperature $T$. The system has Hamiltonian $H$, density matrix $\rho_{i}$, and energy $d E_{\text {sys }}=\operatorname{Tr}\left(\rho_{i} H\right)$. Around the horizon it comes into thermal equilibrium with the black hole and its final state becomes $\rho_{f}=e^{-H / k_{B} T} / Z$. They showed that the total change in entropy can be written as

$$
\delta S \equiv \delta S_{s y s}+\delta S_{B H}=\operatorname{tr}\left[\rho_{i} \ln \rho_{i}-\rho_{f} \ln \rho_{f}\right] \simeq d E_{s y s} / k_{B} T \geq 0 .
$$

Recently, a more accurate derivation of the entropy change is given by Padmanabhan (See chap 4.4 of [17]). Now, we apply his methods to calculate the variation of the entanglement entropy of the local Rindler horizon. Consider a quantum density matrix $\rho_{1}$ of an excited state of a quantum field crossing the Rindler horizon. The density matrix can be obtained by tracing out unobservable modes beyond the horizon. This state has an entanglement entropy $S_{1}=-\operatorname{Tr}\left(\rho_{1} \ln \rho_{1}\right)$. Similarly defined vacuum state $\rho_{0}$ has an entanglement entropy $S_{0}=-\operatorname{Tr}\left(\rho_{0} \ln \rho_{0}\right)$. For the Rindler observer the vacuum state appears to be $\rho_{0}=e^{-H_{R} / k_{B} T_{U}} / Z$, where $H_{R}$ is the corresponding Hamiltonian in the Rindler coordinates and

$$
T_{U}=\frac{\hbar a}{2 \pi k_{B} c}
$$

is the Unruh temperature of the horizon seen by an observer with acceleration $a$. Following [17] one can find that the entanglement entropy difference representing information loss is

$$
\delta S_{E n t} \equiv S_{1}-S_{0} \simeq d E_{\text {sys }} / k_{B} T
$$

in the large acceleration limit. Here, $d E_{\text {sys }} \equiv \operatorname{Tr}\left(\left(\rho_{1}-\rho_{0}\right) H_{R}\right)$. Thus, we can generally use Eq. (1) to calculate the horizon entropy change due to the particle crossing a causal horizon. Based on this result one can guess that the first law applied to causal horizons may have a quantum informational origin and the information loss can be quantified in terms of the entanglement entropy $S_{E n t}$.

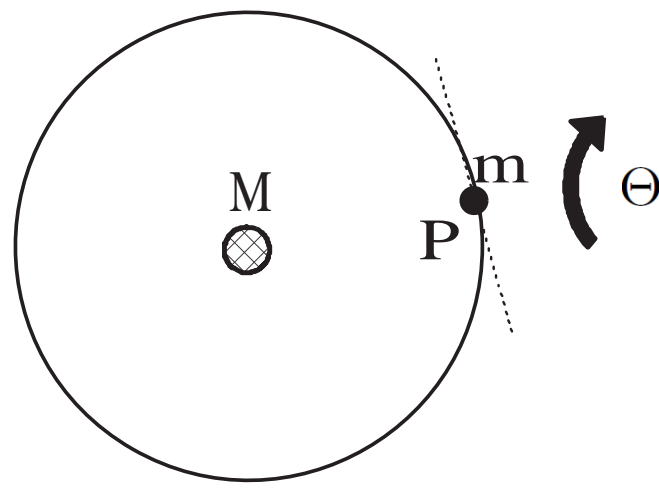

FIG. 1. To calculate the gravitational field at $P$ by a massive object $M$ at the center, consider an accelerating observer $\Theta$ relative to the local inertial frame at $P$, where a small test particle $m$ is. It is possible that for the observer the particle appears to cross a local Rindler horizon (represented by the dotted line) for the observer. This results in the increase of the entropy of the horizon and the Einstein equation describes the energy-information relation at this horizon.

Putting it altogether, now it is natural to imagine that gravity itself has a quantum informational origin. We need to go one more step to confirm that. In 1995, Jacobson showed that one can derive the Einstein equation by 
demanding the first law of thermodynamics,

$$
\delta Q=k_{B} T_{U} d S_{h},
$$

hold at Rindler horizons. Here, $S_{h}$ is the entropy of the horizon and $\delta Q$ is the heat flux crossing the horizon. He also assumed the area law for the horizon entropy. By demanding that the first law holds at local Rindler horizons for each spacetime point, one can derive the Einstein equation. Below we follow his derivation keeping in mind that $d S_{h}=\delta S_{E n t}$ in Eq. (11) or Eq. (5) actually represents the lost information when matter crosses the horizon.

To understand this problem, consider a massive object with mass $M$ at the center and an accelerating observer $\Theta$ with arbitrary large acceleration $a$ relative to the local inertial frame at a point $P$ (See Fig. 1). There is another small test particle $m$ with energy $d E$ experiencing the gravitational field generated by $M$. The bottom line of our new idea is as follows. When the test particle crosses the Rindler horizon, the information erasing and apparent decrease of the total entropy happens to the observer. To save the second law of thermodynamics, the horizon entropy should be increased at least by the amount of the information lost when the matter crosses the horizon. We assume the optimal increase of the entropy. The energy conservation gives $d E_{h}=d E$. Hence, the amount of the entropy change is given by Eq. (1), $d S_{h}=\frac{d E_{h}}{k_{B} T_{h}}=\frac{d E}{k_{B} T_{U}}$. This gives $d E=k_{B} T_{U} d S_{h}$. From this relation at the Rindler horizon one can easily reproduce Verlinde's formalism for entropic gravity (See [18] for details). For example, using $d E \simeq m c^{2}$ and $T_{U}$ one can obtain

$$
d S_{h}=\frac{d E}{k_{B} T_{U}}=\frac{2 \pi c k_{B} m \Delta x}{\hbar},
$$

where $\Delta x \sim 1 / a$ is the horizon distance. This is just the entropy variation formula assumed by Verlinde. It is also justified to invoke the Unruh temperature in his formalism.

\section{EINSTEIN EQUATION FROM INFORMATION LOSS}

In this section, we show that the Einstein equation can be given from information loss and Jacobson's idea relating the gravity to thermodynamics.

Let us study more general situations in the context of the quantum field theory in curved spacetime. Thanks to the equivalence principle one can choose an approximately flat patch for every spacetime point $P$ with boost Killing vector field $\xi_{\alpha}=-\kappa \lambda k_{\alpha}$ generating a local Rindler horizon for the observer $\Theta$ where the matter crosses. Here, $k_{\alpha}$ is the tangent vector to the horizon generators with an affine parameter $\lambda$ and $\kappa$ is the acceleration of the Killing orbit satisfying $T_{U}=\hbar \kappa / 2 \pi k_{B} c$. Then, the local situation in Fig.1 at $P$ can be approximately described using the Rindler coordinate chart in Fig. 2;

$$
\begin{aligned}
c t & =\left(\frac{c^{2}}{a}+X\right) \sinh \left(\frac{a \tau}{c}\right), \\
x & =\left(\frac{c^{2}}{a}+X\right) \cosh \left(\frac{a \tau}{c}\right)
\end{aligned}
$$

where $(t, x)$ is the Minkowski coordinate and $(\tau, X)$ is the Rindler coordinate of the observer. Each point in the figure represents a 2-dimensional spatial plane.

We can now follow Jacobson's derivation and generalize the first law by defining the energy flow across the horizon $\Sigma$

$$
d E=-\kappa \lambda \int_{\Sigma} T_{\alpha \beta} \xi^{\alpha} d \Sigma^{\beta}
$$

where $d \Sigma^{\beta}=\xi^{\beta} d \lambda d A, d A$ is the spatial area element, and $T_{\alpha \beta}$ is the energy momentum tensor of the matter field. Using the Raychaudhuri equation one can denote the horizon area expansion $\delta A \propto d S_{h}$ and the increase of the entropy as

$$
d S_{h}=\eta \delta A=-\eta \lambda \int_{\Sigma} R_{\alpha \beta} \xi^{\alpha} d \Sigma^{\beta}
$$

with some constant $\eta$ [1]. If $S_{h}$ saturates the Bekenstein bound, $\eta=c^{3} / 4 \hbar G$.

It is well known that by partial tracing the Minkwoski vacuum of matter field $\phi$, one can obtain a reduced density matrix $\rho_{0}=e^{-H_{R} / k_{B} T_{U}} / Z$ for the Rindler vacuum, which is a thermal one. (See for example [19].) Here, $Z$ is the 


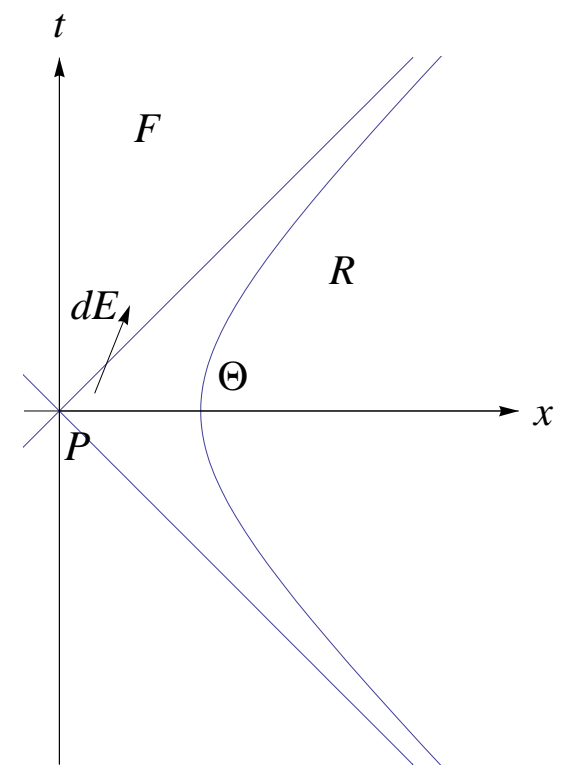

FIG. 2. To calculate the metric evolution at $P$ consider the approximate Rindler chart for the observer $\Theta$, who would see the matter with energy $d E$ crosses the local Rindler horizon. The horizon divides the spacetime into two causally disconnected regions $F$ and $R$, and should expand appropriately to satisfy the Landauer's principle. This leads to the Einstein equation

partition function. This can be done by calculating the wave functional of fields in the left and right patches $\left(\phi_{L}, \phi_{R}\right)$ respectively with the path integral

$$
\psi\left(\phi_{L}, \phi_{R}\right) \propto \int d \phi e^{-S_{E}}
$$

where $S_{E}$ is the Euclidean action. Then, a reduced density matrix is given by $\rho_{R}=\exp \left(-2 \pi H_{R}\right) / Z$, from which one can obtain $\rho_{0}$.

At this moment, if we identify the horizon entropy $S_{h}$ to be the entanglement entropy $S_{E n t}$, we can avoid the use of the holographic principle and some circular logics. Furthermore, in principle one can calculate $\eta$ using the quantum field theory by adding up contributions from all fields [20]. Therefore, we conjecture that the horizon entropy variation connected to Landauer's principle is just $d S_{E n t}$. Although the initial state is not exactly a vacuum state, after the matter crosses the horizon, we can use the entanglement entropy of the vacuum to describe the whole system. This is because, after crossing the horizon, the matter becomes a part of the thermal system (i.e., the vacuum for an inertial observer) beyond the horizon to the outside Rindler observer.

For example, for a spherical horizon with a radius $r$, if there are $N_{j}$ spin degrees of freedom of the $j$-th field, this implies that for a spherical region with radius $r$

$$
S_{E n t}=\sum_{j} \beta_{j} N_{j} \frac{r^{2}}{L_{P}^{2}}=4 \pi \eta r^{2} .
$$

where $\beta_{j}$ is an $O(1)$ numerical constant for the $j$-th field and $L_{P}=\sqrt{8 \pi \hbar G / c^{3}}$ is the reduced Planck length. Srednicki obtained a value $\beta_{j}=0.3$ for the massless scalar field by performing numerical calculations on a sphere lattice. A similar value was obtained for a massless scalar field in the Friedmann universe in [20, 21]. Thus, the Bekenstein bound gives a constrain $\Sigma_{j} \beta_{j} N_{j}=8 \pi^{2}$ on the number and characteristics of quantum fields in the universe [5].

To explain all horizon entropy with $S_{E n t}, N_{j}$ should have a special value. However, despite of this species problem of entanglement entropy, we asserts here that the entanglement entropy is the origin of the horizon entropy needed for thermodynamic gravity models. From the information theoretic viewpoint described above, the entanglement entropy of the vacuum is the most natural candidate for $S_{h}$ related to Landauer's principle. (After completion of the first version of this work, using perturbative quantum gravity, Bianchi indeed showed that the change of entanglement entropy due to the small matter is equivalent to the change of the Bekenstein-Hawking entropy [22]. This result strongly supports our conjecture.)

Inserting Eqs. (8) and (9) into $d E=k_{B} T_{U} d S_{E n t}=\hbar \kappa d S_{E n t} / 2 \pi c$ one can see $2 \pi c T_{\alpha \beta} \xi^{\alpha} d \Sigma^{\beta}=\eta R_{\alpha \beta} \xi^{\alpha} d \Sigma^{\beta}$. For all local Rindler horizons this equation should hold. Then, this condition and Bianchi identity lead to the Einstein 
equation

$$
R_{\alpha \beta}-\frac{R g_{\alpha \beta}}{2}+\Lambda g_{\alpha \beta}=\frac{2 \pi}{\eta c} T_{\alpha \beta}
$$

with the cosmological constant $\Lambda$ as shown in his paper. Results in [22] implies that the conjecture $d S_{h}=d S_{E n t}$ gives the correct value for $\eta$.

\section{DISCUSSIONS}

Strangely, information theoretic aspects of Jacobson's theory were not discussed widely so far, though the entropy $S_{h}$ clearly has an information theoretic meaning in modern physics (See 23]). Now, we can interpret the first law in Eq. (5) in terms of the Landauer's principle. We assumed the second law of thermodynamics is more fundamental than the first one and the first law should be satisfied if the second law holds for all causal horizons blocking information.

There is yet another support for quantum information being the source of entropic gravity. Using Levy's Lemma it was shown that if the universe is sufficiently large and in a generic pure quantum state, a small system entangled with environment in the universe can be effectively thermalized [24].

Our theory reproduces Verlinde's entropic gravity in terms of quantum information. How can one reconcile the irreversibility of the entropy and reversibility of gravity in our interpretation? As in black hole cases, free falling observer comoving with free falling matter would not see the Rindler horizons, while fixed observers (accelerating against the matter frame relatively) can see the horizons. Thus, the entropy in a gravitational system is an observer dependent quantity in general. Nonetheless, since the Einstein equation derived above is covariant, the equation should hold for every frame, once it is satisfied in a specific frame. Therefore, our theory is in concordance with Verlinde's proposal in a general sense.

However, there are some differences between our theory and Verlinde's theory. First, in our theory we assumed neither the proportionality of entropy on the distance, nor the entropic force. The equipartition condition is not necessary either. Second, we suggested the horizon entropy is originated from quantum information erasing at a horizon rather than coarse graining of mysterious microscopic degree of freedom on the horizon. This explain why the derivation of classical gravity is involved with $\hbar$ and why gravity has something to do with entropy or information. The Newton's gravity could arise, of course, from the non-relativistic limit of the Einstein equation. Third, our theory does not demand the generalized holographic principle for equipotential surfaces. Ordinary quantum field theory in curved spacetime is enough to calculate the entanglement entropy of the horizons. In principle, with reasonable assumptions, one may explicitly calculate some relevant physical quantities such as dark energy. Note that we did not assume that the spacetime is emergent but assume the existence of spacetime a priori. Since our theory links quantum mechanics to classical gravity, it might provide us a new way to quantum gravity.

Considering the second law, we expect that the causal horizon area of the universe has a strong tendency to increase [7. That is, matter in the universe distribute themselves so that the horizon entropy of the universe to be maximized. This might be the origin of the gravitational force as an entropic force considered by Verlinde.

Summarizing all these facts, we can say that the Einstein equation simply states that total entropy of matter and horizon should not decrease and this is the bottom line of all gravitational phenomena. The causal structure of the spacetime should be automatically arranged so that the area of the Rindler horizons appropriately increase to compensate the information loss of the matter crossing the horizons.

In short, the Einstein equation links matter to gravity and his famous formula $E=m c^{2}$ links matter to energy. We know also that the Landauer's principle links information to energy. Thus, now we may have a relation between information and gravity, the Einstein equation with the quantum information theoretic interpretation.

\section{ACKNOWLEDGMENTS}

Authors are thankful to Gungwon Kang for helpful discussions. This work was supported in part by Basic Science Research Program through the National Research Foundation of Korea (NRF) funded by the ministry of Education, Science and Technology (2010-0024761), by the Topical Research Program (2010-T-01) of Asia Pacific Center for Theoretical Physics, and by the Korea Research Foundation Grant funded by the Korean Government (MOEHRD, Basic Research Promotion Fund)(KRF-2008-314-C00063), and by the Korea Research Foundation (KRF) grants 
funded by the Korea government (MEST) No. 2010-0011308 (H.K.)

[1] T. Jacobson, Phys. Rev. Lett. 75, 1260 (1995).

[2] C. Eling, R. Guedens, and T. Jacobson, Phys. Rev. Lett. 96, 121301 (2006).

[3] E. P. Verlinde, JHEP 1104, 029 (2011) arXiv:1001.0785 [hep-th]].

[4] T. Padmanabhan, Mod. Phys. Lett. A 25, 1129 (2010) arXiv:0912.3165 [gr-qc]].

[5] J.-W. Lee, J. Lee, and H.-C. Kim, JCAP08(2007)005 hep-th/0701199 (2007).

[6] H.-C. Kim, J.-W. Lee, and J. L. Lee, Mod.Phys.Lett. A25, 1581 (2010), arXiv:0709.3573 (2007).

[7] H.-C. Kim, J.-W. Lee, and J. Lee, JCAP 0808, 035 (2008).

[8] J.-W. Lee, J. Lee, and H.-C. Kim, Proceedings of the National Institute for Mathematical Science 8, 1, arXiv:0709.0047 [hep-th] (2007).

[9] J.-W. Lee, H.-C. Kim, and J. Lee, Mod. Phys. Lett. A25, 257 (2010).

[10] M. Li and Y. Wang, arXiv:1001.4466 (2010).

[11] Y. Zhang, Y.-g. Gong, and Z.-H. Zhu, arXiv:1001.4677 (2010).

[12] S.-W. Wei, Y.-X. Liu, and Y.-Q. Wang, arXiv:1001.5238 (2010).

[13] D. A. Easson, P. H. Frampton, and G. F. Smoot, Int. J. Mod. Phys. A 27, 1250066 (2012) arXiv:1003.1528 [hep-th]].

[14] M. Li, Phys. Lett. B 603, 1 (2004).

[15] M. Srednicki, Phys. Rev. Lett. 71, 666 (1993).

[16] D. D. Song and E. Winstanley, Int. J. Theor. Phys. 47, 1692 (2008).

[17] T. Padmanabhan, arXiv:0911.5004 (2009).

[18] J. -W. Lee, Found. Phys. 42, 1153 (2012) arXiv:1003.4464 [hep-th]].

[19] L. Susskind and J. Lindesay, An introduction to black holes, information and the string theory revolution: The holographic universe, World Scientific (2005).

[20] R. Müller and C. O. Lousto, Phys. Rev. D 52, 4512 (1995).

[21] M. Srednicki, Phys. Rev. Lett. 71, 666 (1993).

[22] E. Bianchi, arXiv:1211.0522 [gr-qc].

[23] G. Chirco and S. Liberati, Phys. Rev. D 81, 024016 (2010) arXiv:0909.4194 [gr-qc]].

[24] S. Popescu, A. J. Short and A. Winter, Nature Physics 2, 754 (2006) 